\title{
Liquefaction of lignin using chemical decomposition and its application to polyurethane foam
}

\author{
Jisu Jeong, ${ }^{1}$ Woo Sik Kim, ${ }^{2}$ Min Wook Lee, ${ }^{3}$ Munju Goh ${ }^{1, *}$ \\ 1 Department of Chemical Engineering., Konkuk University, Gwangjin, Seoul 05029, Republic of \\ Korea \\ 2 Fibrous Ceramics \& Aerospace Materials Center, Korea Institute of Ceramic Engineering \& \\ Technology, Jinju-si, Gyeongsangnam-do 52851, Republic of Korea \\ 3 Institute of Advanced Composite Materials, Korea Institute of Science and Technology, Chudong-ro, \\ Bongdong-eub, Jeonbuk 55324, Republic of Korea
}

Corresponding author e-mail: mgoh@konkuk.ac.kr 
Solvent : $\mathrm{D}_{2} \mathrm{O}$

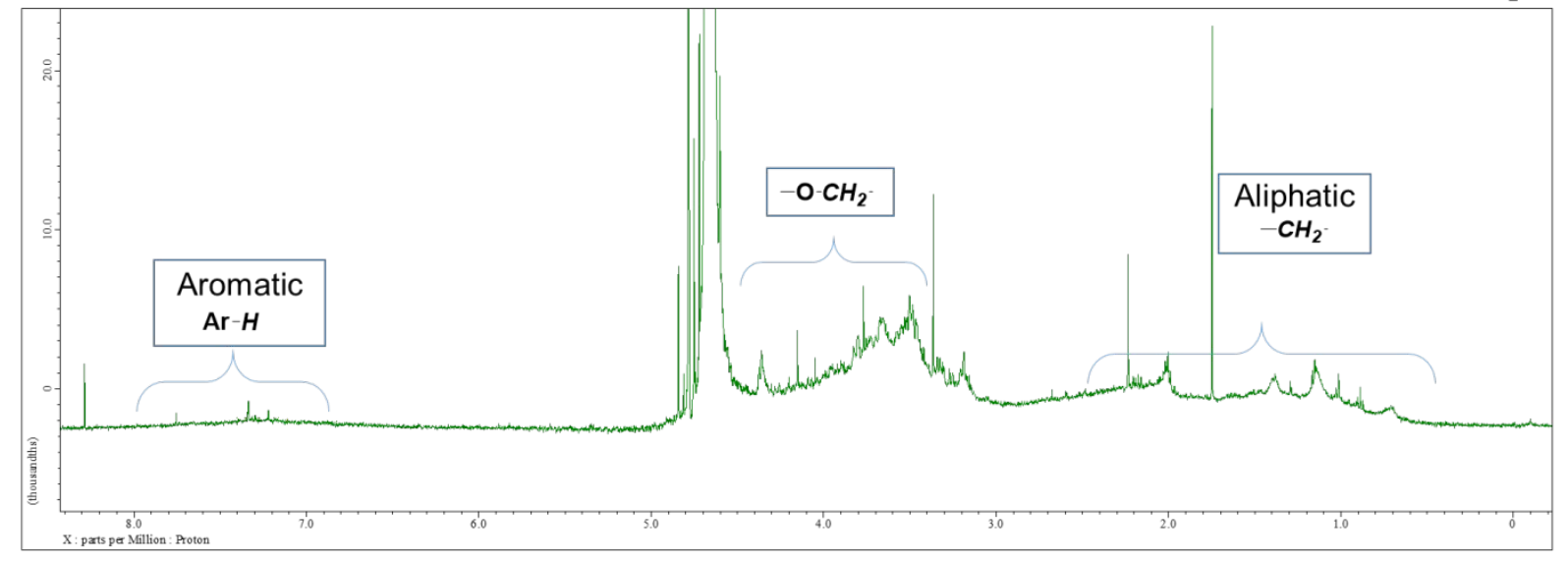

Figure S1. NMR result of 1-LN with $\mathrm{D}_{2} \mathrm{O}$ solvent.

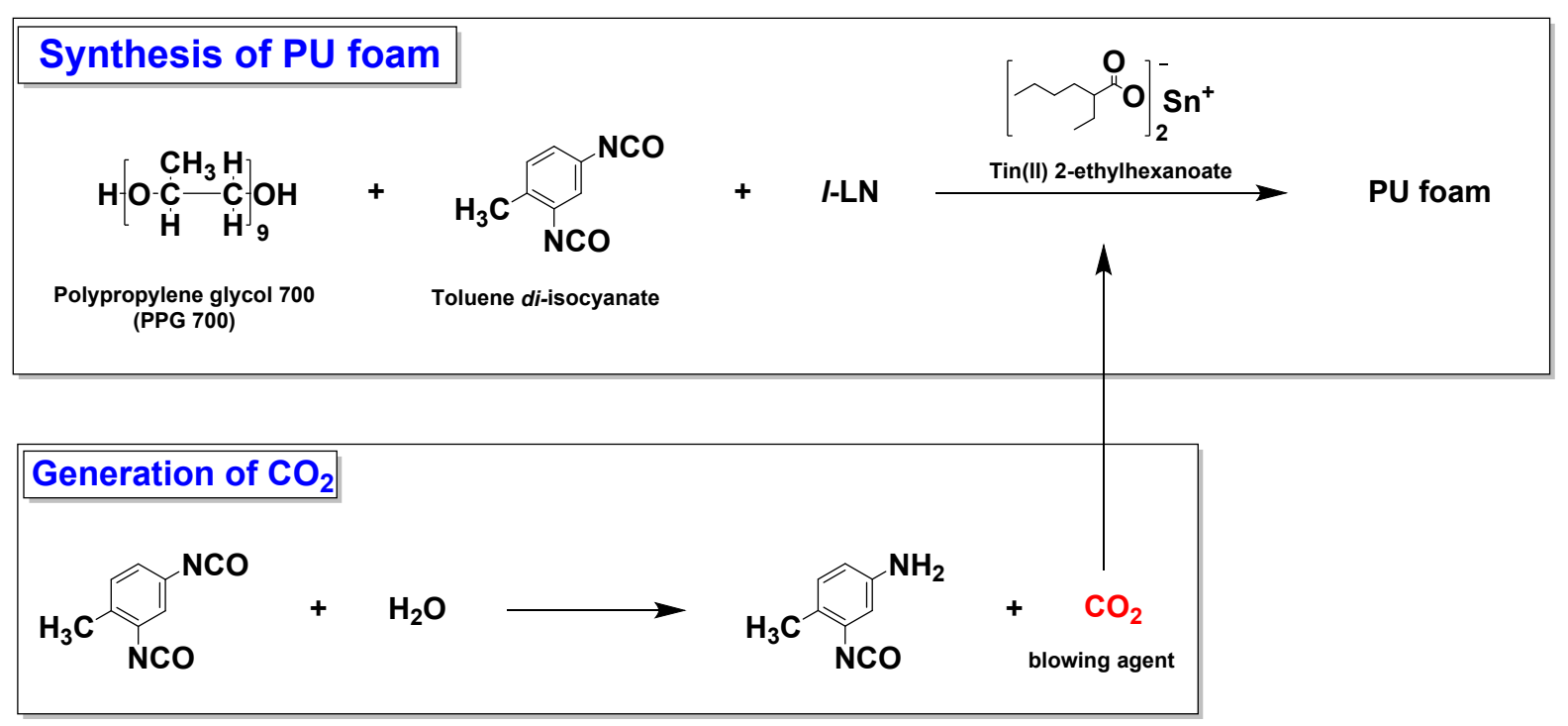

Scheme S1. Synthetic routes for PUF. 
(a)

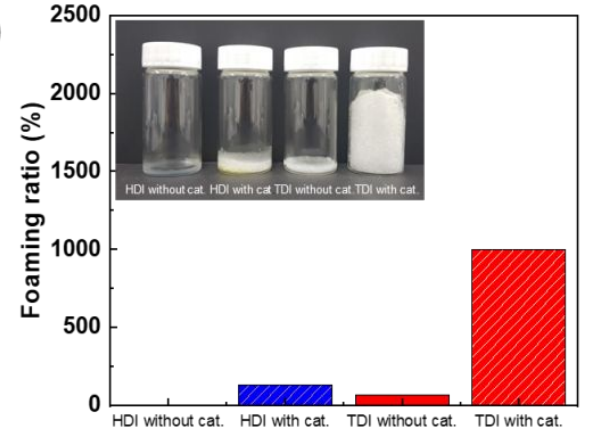

(c)

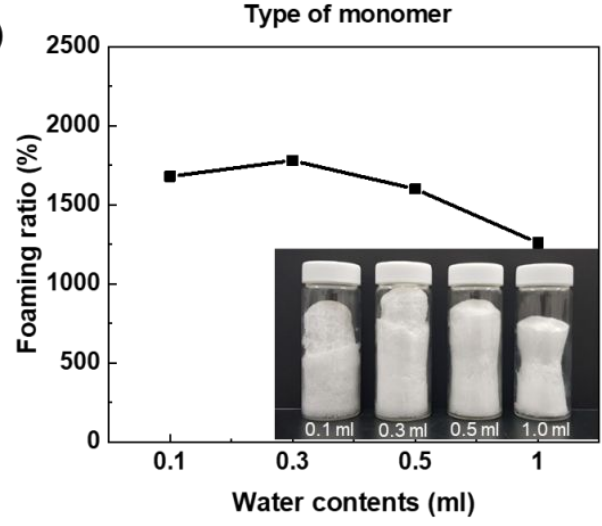

(b)

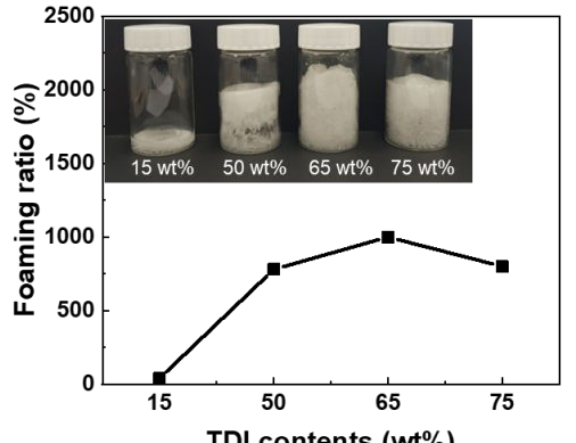

(d)

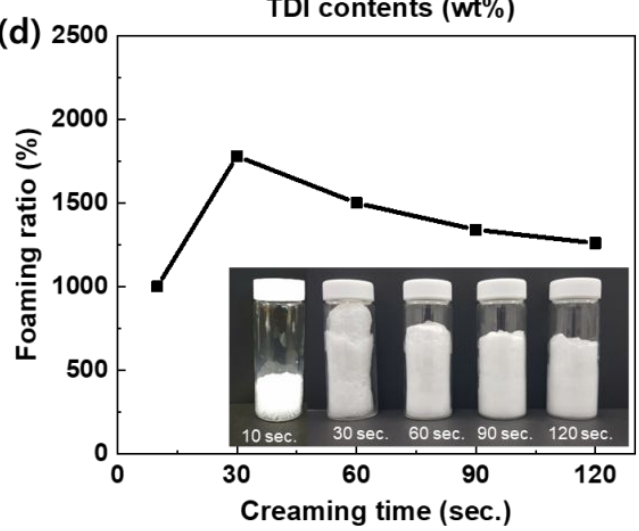

Figure S2. Variation of foaming ratios (a) by changing the types of monomers (aliphatic or aromatic diisocyanate were used), and presence or absence of catalyst, (b) amount of diisocyanate monomer, (c) water contents, (d) creaming time. 

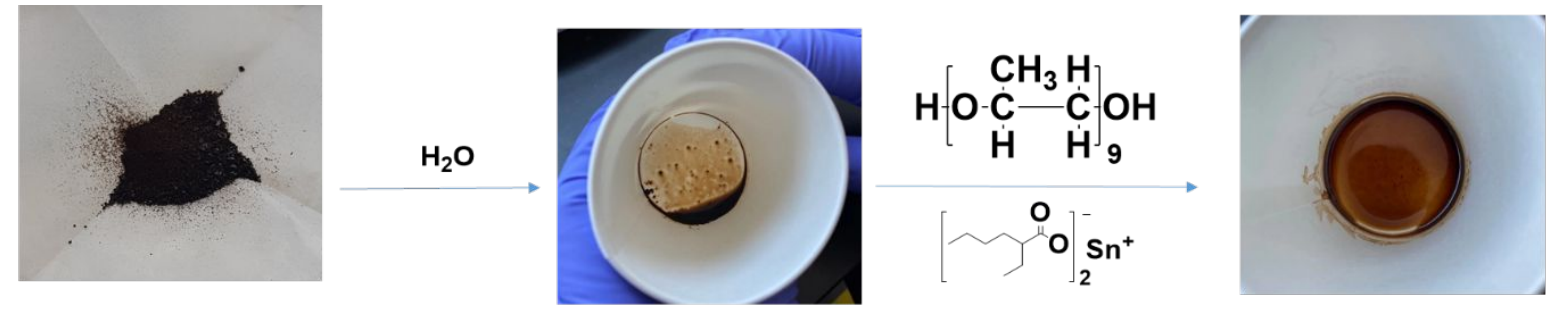

I-LN powder

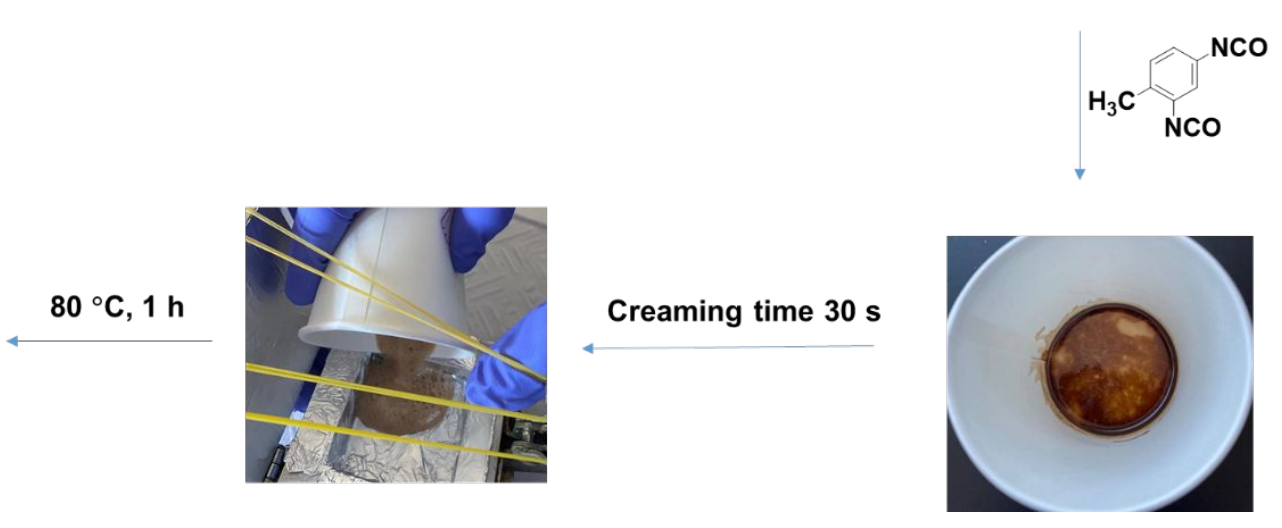

Figure S3. Synthesis procedure of $l$-LN containing PUFs.

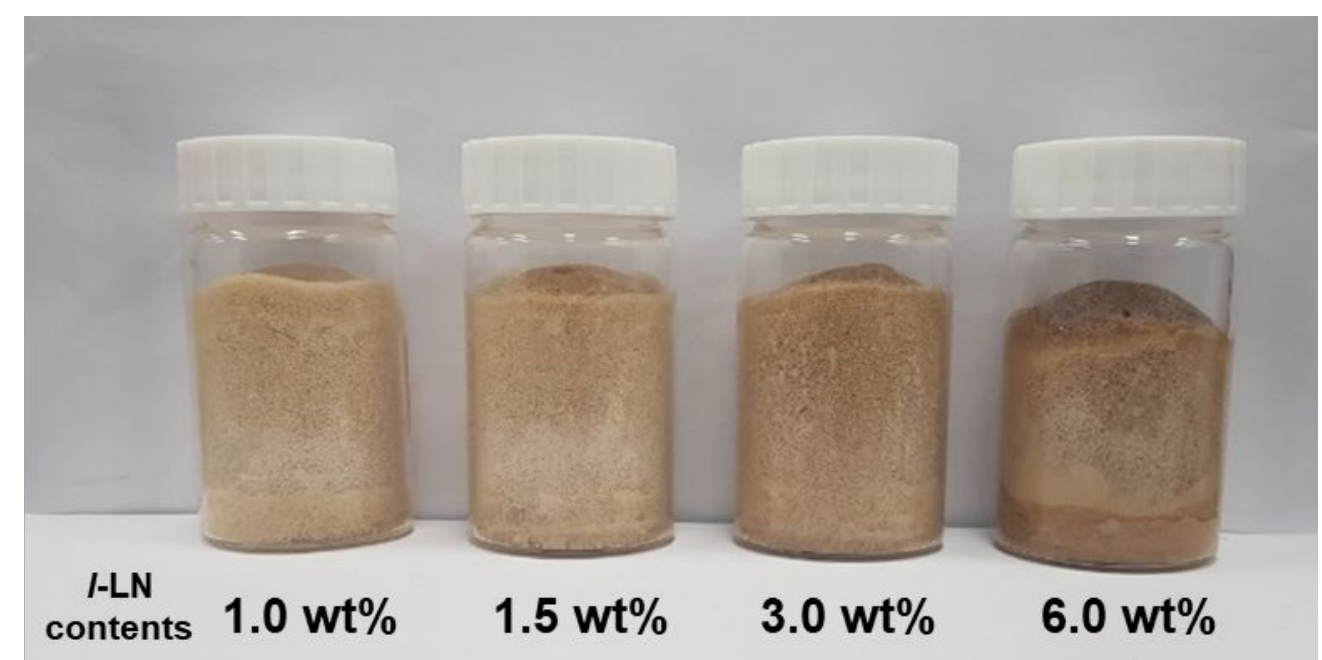

Figure S4. Photographs of 1-LN containing PUFs. 


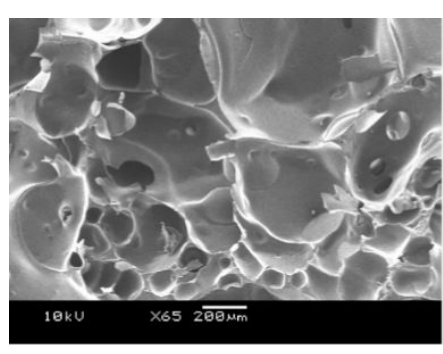

I-LN (0 wt\%)

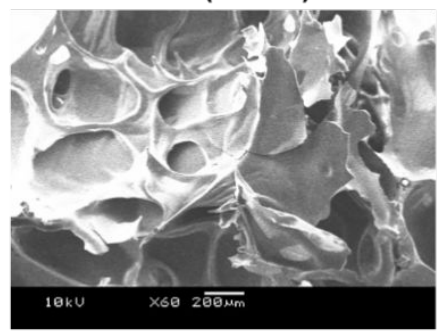

I-LN (3 wt\%)

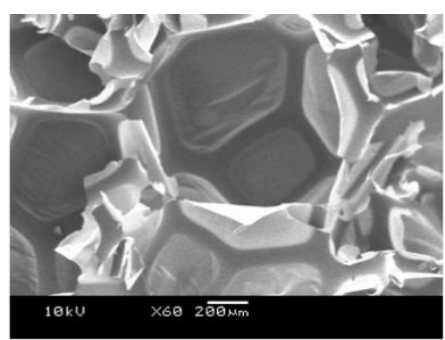

I-LN (1 wt\%)

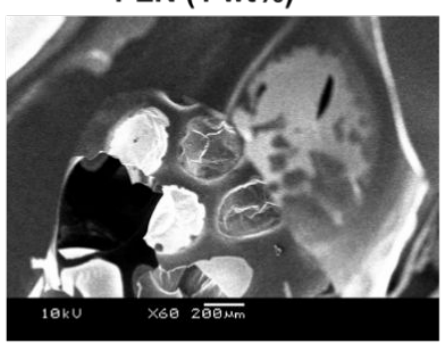

I-LN (6 wt\%)

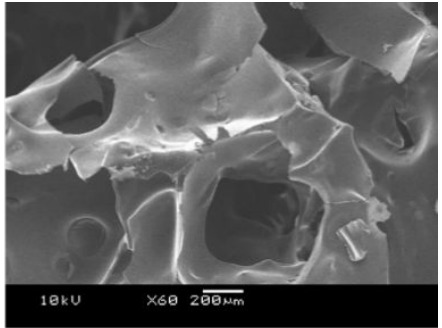

I-LN (1.5 wt\%)

Figure S5. SEM results of cell structured $l$-LN containing PUFs. 